\title{
Long-lasting aftereffect of brief prism exposure
}

\author{
STUART T. KLAPP*, SARAH A. NORDELL, KATHY C. HOEKENGA, and CAROL B. PATTON \\ California State University. Hayward, California 94542
}

\begin{abstract}
The aftereffect of $15 \mathrm{~min}$ of active adaptation to wedge prism displacement was shown to persist for as long as 2 weeks.
\end{abstract}

The coordination of visually guided hand movements can readily be modified by practice in moving the hand while the visual axis is displaced laterally by a wedge prism. After a few minutes of such practice, an adaptation occurs which can be observed as (1) reduction in the error in pointing at a target viewed through the prism (prism error), and as (2) an error in the opposite direction (the aftereffect) which appears when $S$ attempts to point at a target viewed without the prism. In the course of attempting to replicate some of the standard findings in this paradigm, the authors tested Ss in different conditions spaced 2 days apart. This was done as a matter of convenience, with the assumption that the effect of prior training would dissipate during the intervening days as a result of practice with hand movements in everyday life. However, it appeared that this was not the case. Informal observations suggested that target-pointing errors had been biased by the effect of a few minutes of exposure to prisms 2 days earlier. The present study represented a systematic attempt to replicate this informal observation.

\section{METHOD}

\section{Subjects}

The Ss were right-handed students reporting no known history of eye-movement therapy or prismatic correction, who participated in order to fulfill a requirement in introductory psychology at California State University, Hayward. Alternate Ss were assigned to base-left or base-right prisms in the order in which they reported for the experiment. Data from one $S$ in the base-right group were discarded due to an extreme initial error in pointing position which did not shift after prism exposure. After data from this $S$ were discarded, there remained 9 Ss with base-right prisms and 10 with base-left prisms.

\begin{abstract}
Apparatus and Procedure
The 10-diopter wedge prisms were mounted on welder's goggles, with the prism over the right eye and vision from the left eye occluded by black tape. Testing and adaptation were carried out while $S$ sat in front of a frame which supported a target comprising a heavy black vertical line on a white background. The S's right eye and right shoulder were centered with respect to the target, which was $65 \mathrm{~cm}$ from the eye. For adaptation training, the $S s$ repeatedly pointed at this target line, using a thrust of the right arm and hand away from the body and toward the target. For testing, Ss pointed with the same motion, but vision of the arm and hand was occluded by a horizontal
\end{abstract}

*Requests for reprints should be sent to Stuart T. Klapp, Department of Psychology, California State University, Hayward, California 94542. cover above the plane of the hand movements. The target line extended above the cover and remained in view. No feedback was provided during or after the testing sessions.

Design

All measurements were based on the mean of the errors of pointing to target from four successive attempts. An initial error measurement was obtained in this way prior to any prism exposure. Then the prism goggles were positioned, and a preadaptation prism error was obtained. After $1 \mathrm{~min}$ of adaptation, the pointing error was obtained, first with prism positioned (prism error), then without prism (aftereffect). This was followed by 14 additional 1 -min adaptation sessions spaced by $1.5-\mathrm{min}$ rest intervals. No further test measurements were obtained until the conclusion of the last adaptation session, when the prism error and aftereffect were again measured. The latter measurement became the first of a series of tests of the aftereffect measured after various intervals of normal activity. The intervals selected included, in addition to the above initial measurement, the following cumulative postadaptation times: $5 \mathrm{~min}, 15 \mathrm{~min}, 30 \mathrm{~min}, 45 \mathrm{~min}, 60 \mathrm{~min}$, and 1 day. Measures were also obtained from 16 (of the 19) Ss after 2 days, and from 17 Ss after intervals ranging from 2 to 4 weeks. The Ss for whom these long-interval data were not available failed to respond to the request to appear for further testing.

\section{RESULTS AND DISCUSSION}

The mean of the initial pointing errors was $0.9 \mathrm{deg}$ for the 19 usable Ss. The data to be reported (Fig. 1) were normalized individually for each $S$ by subtracting that $S$ 's initial bias from each observed pointing position. All statistical tests based on error magnitude involved comparison of initial pointing position to the position at the interval in question. Data from base-left and base-right Ss were combined, since there was no significant effect of prism orientation on the magnitude of the aftereffect.

Prism error and aftereffect were observed initially and after $1 \mathrm{~min}$ and $15 \mathrm{~min}$ of adaptation. As is apparent in Fig. 1, both measures showed a strong and rapid adaptation. Prism error successively decreased as adaptation proceeded, $F(2,34)=175, p<.001$, and the aftereffect showed a corresponding increase, $F(2,34)=$ $37.4, p<.001$.

As is clear from Fig. 1, aftereffect decreased as a function of postadaptation time, $F(6,102)=6.5$, $\mathrm{p}<.001$ (through the 1-day interval, all $19 \mathrm{Ss}$ ). However, the major finding of interest is the fact that some aftereffect persisted even after quite long intervals. 


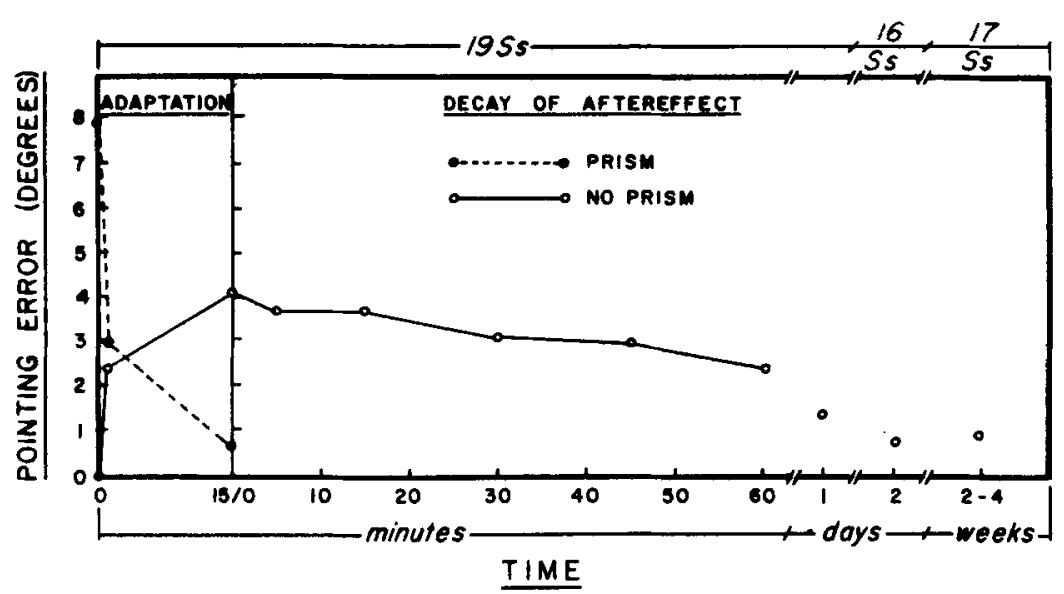

Fig. 1. Adaptation and decay of aftereffect.

The aftereffect was apparent at the 1-day interval for 16 of the 19 Ss $(p<.01$ by sign test $)$ and displayed a significant shift from the initial error, $F(1,17)=11.3$, $p<.01$. The aftereffect still was significant at the 2-day interval, $F(1,14)=5.8, p<.05$, and at the 2- to 4-week interval, $F(1,15)=8.0, p<.05$. However, data at these two longer intervals were incomplete due to the failure of some Ss to return, and the aftereffect was significant only when assessed by the parametric $F$ test and not by sign test.

The only other study of decay of prism aftereffect which appears to be directly comparable to the present experiment is that of Hamilton and Bossom (1964), who investigated only the first $15 \mathrm{~min}$ of recovery. They observed a 50\% reduction in aftereffect when Ss sat passively in the dark ("nonreafferent" condition), compared to a loss of only $10 \%$ over the same period of time in the present experiment. Since our Ss could engage in normal visual-motor activity during the intervening period, one might expect that, if anything, our Ss should have shown a faster rather than a slower rate of decay. One possible explanation which would account for this comparison deals with the timing of the adaptation training. Our Ss received $15 \mathrm{~min}$ of practice, which was spaced into $1-\mathrm{min}$ intervals with intervening rest, while Hamilton and Bossom used 15 min of massed practice. If the general principles of learning are assumed to apply to prism adaptation, one would expect more resistance to forgetting after the spaced practice, as in the present experiment. Another factor which might also account for the greater persistence in the present experiment is the nature of the hand movements. In the present experiment, a thrusting movement was involved, while Hamilton and Bossom employed a lateral movement. Both Baily (1972) and Freedman (1968) have reported that these procedures are not equivalent with respect to the aftereffect.

\section{REFERENCES}

Baily, J. S. Adaptation to prisms: Do proprioceptive changes mediate adapted behavior with ballistic arm movements? Quarterly Journal of. Experimental Psychology, 1972, 24, 8-20.

Freedman, S. J. Perceptual compensation and learning. In S. J. Freedman (Ed.), The neuropsychology of spatially oriented behavior. Homewood, Ill: Dorsey Press, 1968.

Hamilton, C. R., \& Bossom, J. Decay of prism aftereffects. Journal of Experimental Psychology, 1964, 67, 148-150.

(Accepted for publication December 28, 1973.) 